\title{
The effect of a preanaesthesia clinic consultation on adult patient anxiety at a tertiary hospital in Kenya: a cohort study.
}

\author{
Anthony Kamau', Vitalis Mung'ayi ${ }^{1}$, Gerald Yonga ${ }^{2}$
}

1. Department of Anaesthesia, Aga Khan University, East Africa, Nairobi, Kenya.

2. Department of Internal Medicine, Aga Khan University, East Africa, Nairobi, Kenya.

\begin{abstract}
Background: Preoperative anxiety is a common perioperative complication seen in approximately $11-80 \%$ of adults undergoing surgery. One of the goals of the preanaesthesia clinic is to allay anxiety. A preanaesthesia clinic evaluation has been shown to reduce anxiety however current studies on anxiety and the preanaesthesia clinic have not quantified this reduction.

Objective: To determine the reduction in anxiety in patients evaluated in the clinic versus those evaluated in the ward.

Methods: Fifty one adult patients with 28 patients in anaesthesia clinic (AC) group and 23 in the ward (W) group were sequentially recruited from both the surgical, gynaecology and antenatal outpatient clinics and the wards. The patient's State Trait Anxiety Index (STAI) was taken once the patient was booked for theatre. The patients then had a preanaesthesia evaluation either in the preanaesthesia outpatient clinic (PAC) or in the wards. Another STAI score was taken in the preoperative area in theatre on the day of surgery. The difference in the change of STAI scores in both groups was then analysed.

Results: Fifty one adult patients were recruited with 28 in the AC group and 23 in the W group. The majority of patients were female $(n=38)$. Statistically significant difference was seen in the reduction of the anxiety scores between the clinic group 2.143 (C.I $=1.384-2.902)$ and ward group $0.74(\mathrm{C} . \mathrm{I}=0.17-1.31)$ with a $\mathrm{p}$ value $=0.0051$. There was also significant difference in reduction in anxiety scores within ward group in the patients with no prior anaesthetic experience having a greater reduction than those with prior anaesthetic experience. There were no other significant differences between the two groups.

Conclusion: Patients evaluated in the anaesthesia clinic had a greater reduction in their anxiety but it was not as much as hypothesised which may be due to the multi-factorial nature of preoperative anxiety. A larger multicenter study is recommended to increase generalizability to the population.

Keywords: Preanaesthesia clinic, consultation on adult patient anxiety, cohort study, Kenya.

DOI: https://dx.doi.org/10.4314/ahs.v17i1.18

Cite as: Kamau A, Mung'ayi V, Yonga G. The effect of a preanaesthesia clinic consultation on adult patient anxiety at a tertiary hospital in Kenya: a cohort study. Afri Health Sci. 2017;17(1): 138-147. https://dx.doi.org/10.4314/abs.v17i1.18
\end{abstract}

\section{Introduction}

Anxiety is one of the most common perioperative complications of anaesthesia for surgical procedures. It is a psychological state that has many definitions according to different literature sources available.

It is defined by Spielberger et $\mathrm{al}^{1}$ as " a subjective reaction consisting of feelings of tension, apprehension, nervousness, and worry, and activation of the autonomic nervous system." According to Tyrer et $\mathrm{al}^{1}$ anxiety is
Corresponding author:
Vitalis Mung'ayi,
Department of Anaesthesia,
Aga Khan University, Nairobi, Kenya.
Email: vitalis.mung’ayi@aku.edu,
mungayi@gmail.com

defined as "a state of mood or emotions of which an excess is unpleasant; it is concerned with uncertainty and is directed towards the future rather than the past."

Current literature on the prevalence of preoperative anxiety done in Western countries and in Asia estimates that between $11-80 \%$ of all surgical patients experience preoperative anxiety ${ }^{2-6}$. Several factors predispose patients to preoperative anxiety. History of cancer and smoking, psychiatric disorders, negative future perception, moderate to severe depressive symptoms, high trait-anxiety, moderate to intense pain, medium surgery, female gender, ASA category III, up to 12 years of education and more than 12 years of education constitute independent risk factors for preoperative state-anxiety. ${ }^{7}$

Preoperative anaesthesia assessment clinics have been introduced over the last 3 decades and are becoming increasingly important in the delivery of efficient and cost 
effective services to surgical patients. The use of a preanaesthesia clinic was suggested as early as 1949 by J A Lee as cited by Lew et $\mathrm{al}^{9}$. There has been a steady increase in ambulatory surgery worldwide. This has led to an increased demand for outpatient preanaesthesia evaluation services. PAC offers various advantages which include a reduction in preoperative testing, subspecialty consults, cancellation rates and confers an economic benefit. ${ }^{8-11,19}$

Disadvantages of the preoperative clinic may include additional consultation costs and longer hospital waiting times after surgical consultation.

According to the American Society of Anaesthesiologists, one of the goals of the PAC is to allay anxiety ${ }^{20}$. There are few PAC's on the continent and only one in Eastern Africa and as such there is a scarcity of studies on preoperative anxiety from the African continent and modes of managing this problem are primarily drawn from western practices.

Anxiety was described in 2 forms by Spielberger et $\mathrm{al}^{12}$. State anxiety is "a temporal cross section in the emotional stream of life of a person consisting of subjective feelings of tension, apprehension, nervousness and worry and activation (arousal) of the autonomic nervous system." Trait anxiety is "the relatively stable individual differences in anxiety proneness (i.e. between people in the tendency to perceive stressful situations as dangerous or threatening and in disposition to respond to such situations with more frequent and intense elevations in state anxiety)."

Preoperative anxiety is prior to a surgical procedure. There is no classification of preoperative anxiety in the Diagnostic and Statistical Manual of Mental Disorders (DSM) IV criteria ${ }^{23}$ as only "specific phobias" e.g. needle phobia are categorised. Preoperative anxiety may include both known phobias and unknown threats or fears.

There are many different adverse effects of anxiety on the body ${ }^{13}$. Physiological effects include tachycardia, hypertension, elevated temperature, sweating, increasedmuscle tone, abdominal pain, nausea, pallor, reduced digestive and immune functions, urgency and a heightened sense of touch, smell or hearing. These are usually as a result of stimulation of the sympathetic nervous system. Psychological effects include tiredness, feelings of threat, reduced self-esteem, and withdrawal from interpersonal relations, cognitive disorders, increased tension, apprehension, nervousness and aggression. The behavioural symptoms involve motor anxiety, pointless activities, frequent position changes, twitches.
STAI has been found to have positive correlations with other scoring systems, namely Multiple Affect Adjective Checklist(MACCL), Edwards Personal Preference Scale(EPPS), Minnesota Multiphasic personality Inventory, Cornell Medical Index and Army Beta Intelligence Test. $^{12}$

There is little evidence in the current literature suggesting the best timing of a preanaesthesia review. An observational study by Pollard et al done to investigate if there is a difference in cancellation rates between a preoperative evaluation done a day before surgery and a preoperative evaluation done 2 to 30 days prior to surgery concluded that there was no difference in cancellation rates between the two groups. ${ }^{10}$

According to the American Society of Anaesthesiologists "A preanaesthesia evaluation is considered a basic element of anaesthesia care" 17 . Preanaesthesia evaluation can be described as " the process of clinical assessment by an anaesthetist, which precedes the delivery of anaesthesia care for surgery and non surgical procedures." ${ }^{17}$

There are currently no studies that have quantified the reduction in anxiety levels due to a standard PAC consultation. The difference between the mean anxiety of the general population and clinically significant anxiety is 10 points ${ }^{18}$. Therefore a difference in 10 points between the 2 groups was chosen as being clinically significant.

Studies so far have been inconclusive regarding the optimal time for a preanaesthesia review. It has been advised that this should be guided by "patient demographics and clinical conditions, the type and invasiveness of the procedure and availability of resources provided by the specific practice environment."'. The current hospital policy on informed consent states that the validity of consent expires after 14 days. Therefore patients seen after this period were excluded from the study.

This study aimed to establish the effectiveness of a preanaesthesia evaluation done 48 hours prior to surgery in the PAC in reducing preoperative anxiety in comparison to a standard anaesthesia preoperative evaluation done in the ward, the day before surgery. The STAI form which is a validated tool for measurement of preoperative anxiety was used to quantify anxiety before and after the intervention.

Our research question was: does a standard preoperative evaluation in the outpatient clinic evaluation performed at least 48 hours prior to surgery reduce the STAI score by at least 10 points when compared to the standard pre- 
operative evaluation done on the day before surgery in the ward?

The aim of the study was to assess whether the standard preanaesthesia evaluation in the outpatient clinic would reduce the preoperative anxiety by 10 points as assessed by the STAI score when compared to a standard preoperative evaluation in the ward.

\section{Methods}

The study was performed following approval from the research and ethics review committee at the Aga Khan University, East Africa. It was a cohort study and before - after study. The study was conducted at Aga Khan University Hospital, Nairobi. Patients were recruited from the surgical outpatient, gynaecological outpatient, antenatal clinics and the wards.

The sample population was all patients going for elective non cardiac surgical anaesthetic procedures during the study period.

All surgical patients attending the pre-anaesthesia clinic at least 48 hours prior to elective surgery and all patients having a preanaesthesia evaluation in the wards a day prior to elective surgery aged above 18 years were included in the study.

\section{Reasons for exclusion from the study were:}

1. Patients seen in the anaesthesia clinic more than 2 weeks before elective surgery.

2. Patients seen in the ward more than 24 hours prior to elective surgery.

3. Patients undergoing surgical procedures due to malignancy.

4. Patients undergoing cardiac surgery.

5. Patients with diagnosed psychiatric illness.

6. Patients undergoing neurosurgery for ailments that may cause changes in cognitive function.
7. Patients, who cannot understand, read or write high school level English.

8. Inability to understand the consent due to language barrier, inability to read or write.

9. Refusal to participate in the study.

The sample size formula was selected to show a 10 points more in reduction of the STAI score of patients evaluated in the outpatient PAC compared to those patients who were evaluated in the ward. The difference of 10 points chosen was the same as that seen to distinguish between the mean anxiety scores of the general population from the anxiety scores clinically anxious patients (14).

For the purpose of sample size calculation $\mathrm{p}$ - Value $=$ 0.05 was considered significant with a Power $=90 \%$. Standard deviation $=10$ this value was chosen because it was the same that Spielberger et al who validated the use of the STAI form ${ }^{14}$.

Fifty one adult patients were recruited with 28 in the anaesthesia clinic group and 23 in the ward group. We gave all potential participants oral and written explanationon the purpose and procedure of the study and a signed informed consent was obtained. Recruitment was done in a sequential manner. No randomization was done for this study.

Once consent was given the patient completed a self administered questionnaire which included the pre-anaesthesiaevaluation STAI form and the demographic data of the patient.

The patients in the clinic group then had a standard preanaesthesia evaluation at least 48 hours prior to surgery according to the hospital designed preanaesthesia chart. This evaluation was done by either consultants or residents who ran the PAC on different days of the week. For the patients in the ward group they received a preanaesthesia evaluation a day before surgery that was similar to the one performed in the anaesthesia clinic i.e. according to the preanaesthesia chart. Patient flow diagram is shown in Figure 1. 


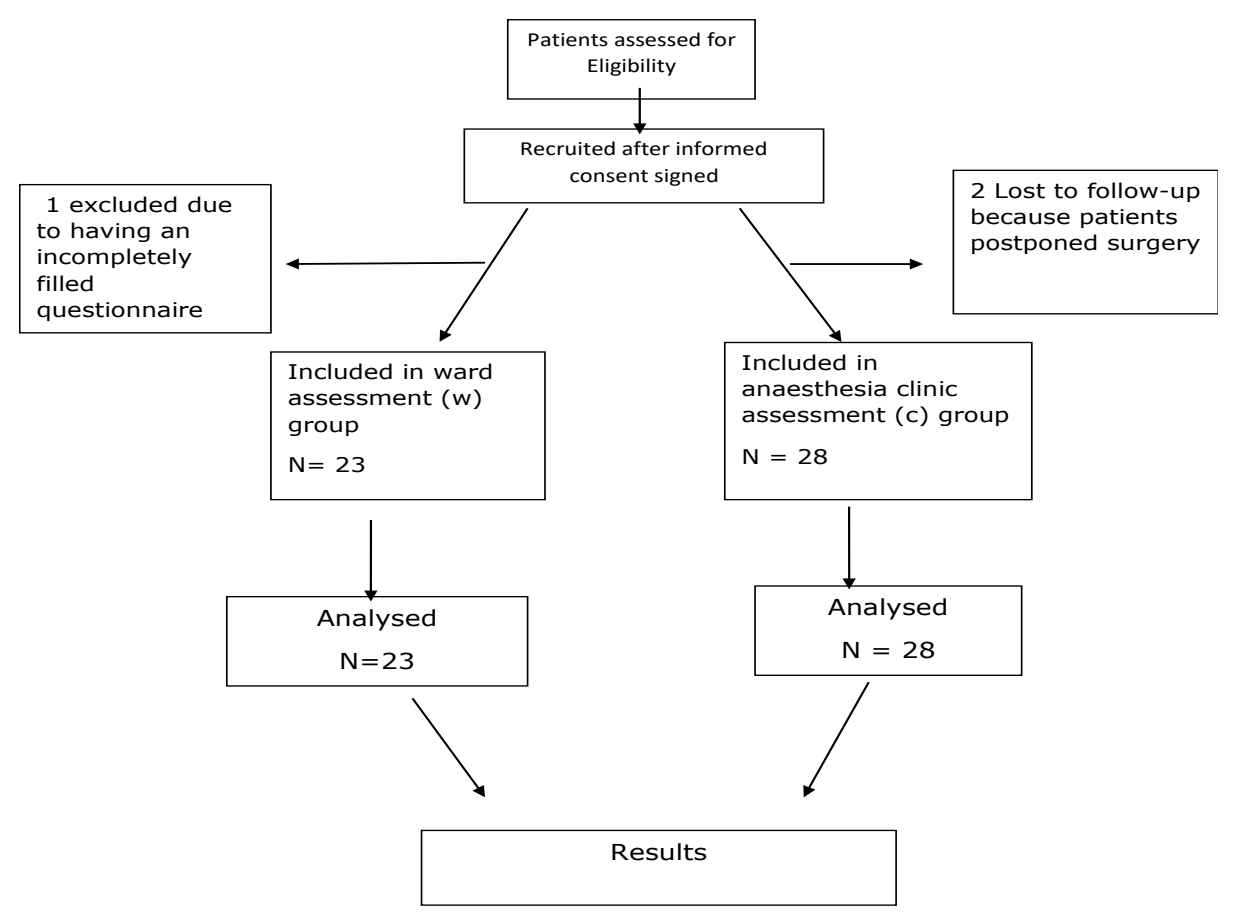

The post evaluation STAI forms were then administered to all the study participants in the preoperative area of the operating theatres. The Post evaluation STAI form were administered a minimum of 12 hours (i.e. night before surgery) after the pre-evaluation STAI form for patients evaluated in the ward and a minimum 48 hours for the patients seen in the clinic. After the forms were filled the data was be collected and the results analysed.

Upon collection data was entered into the statistical software (SPSS version 15) on the same day in a coded form and saved, awaiting analysis. All data entered was verified by the principal investigator. In the case of missing data the principal investigator conducted a follow up and collected the missing data from the patient or from the patient medical records. Every precaution was taken to respect the privacy of the patients whose data was collected and analysed in this study. Patient data was identified by a unique identifier number. However, in the course of monitoring data quality and adherence to the study protocol only the study supervisors referred to the recruited patient's medical records; after analysis the data was stored in soft copy with the research support unit.

The baseline pre-anaesthesia evaluation scores were compared to post-evaluation scores within groups and also comparison of both pre evaluation and post evaluation scores between the 2 groups was done. The relative risk difference between the 2 groups was also determined. In addition the relative risks differences according to gender, level of education and type of surgery was determined. The baseline demographics were analysed and comparisons done between the two groups Analysis was performed using the SPSS statistical software with the help of a statistician who had been involved from the initial development of the study as percentages, means $+/$ - standard deviation unless otherwise specified. The means of change in anxiety score within and between groups was compared using t-test. P- Values $\leq 0.05$ were considered statistically significant. Linear regression was used to establish the relationship between the risk factors i.e. gender and age, with change in anxiety.

In case a patient was noted to have clinically significant levels of anxiety according to the STAI score on the morning of surgery, the interviewer was permitted to intervene. The interviewer reassessed the patients to ascertain the source of the anxiety so that further counselling and appropriate management was undertaken to manage the anxiety including recommending to the anaesthetist performing the procedure to premedicate the patient with an anxiolytic drug.

The patient was free to withdraw from the study at any stage and was still accorded standard care. 
Figure 2: Bar chart showing recruitment by sex at study settings

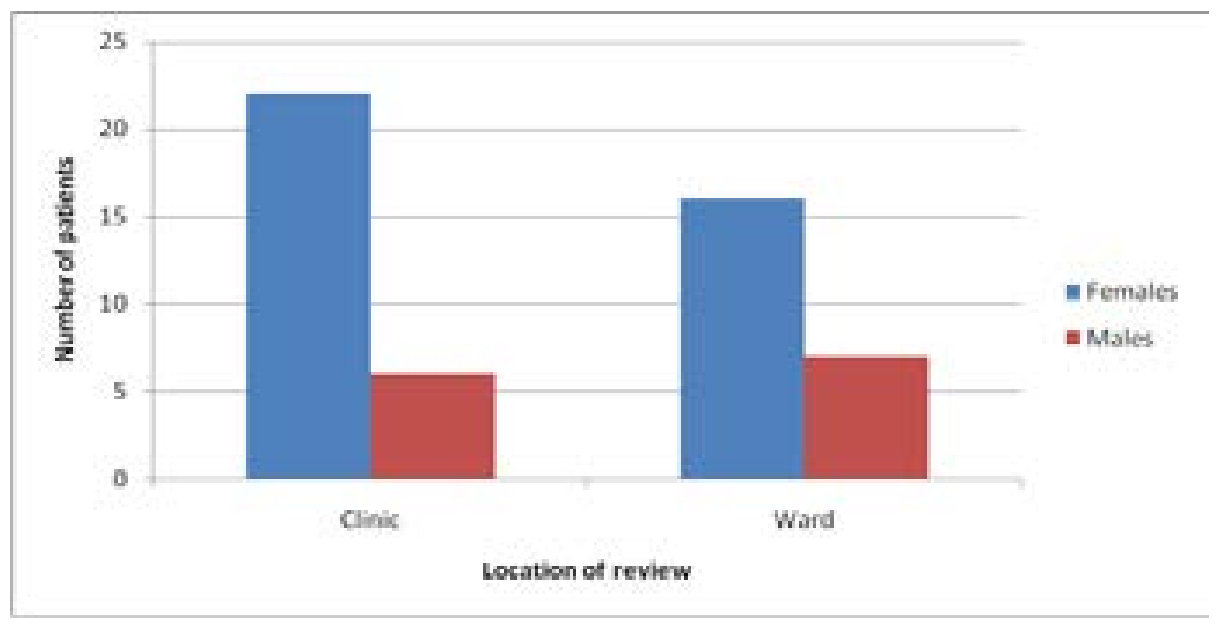

Figure 3: Bar chart representing surgical procedures planned for recruited patients

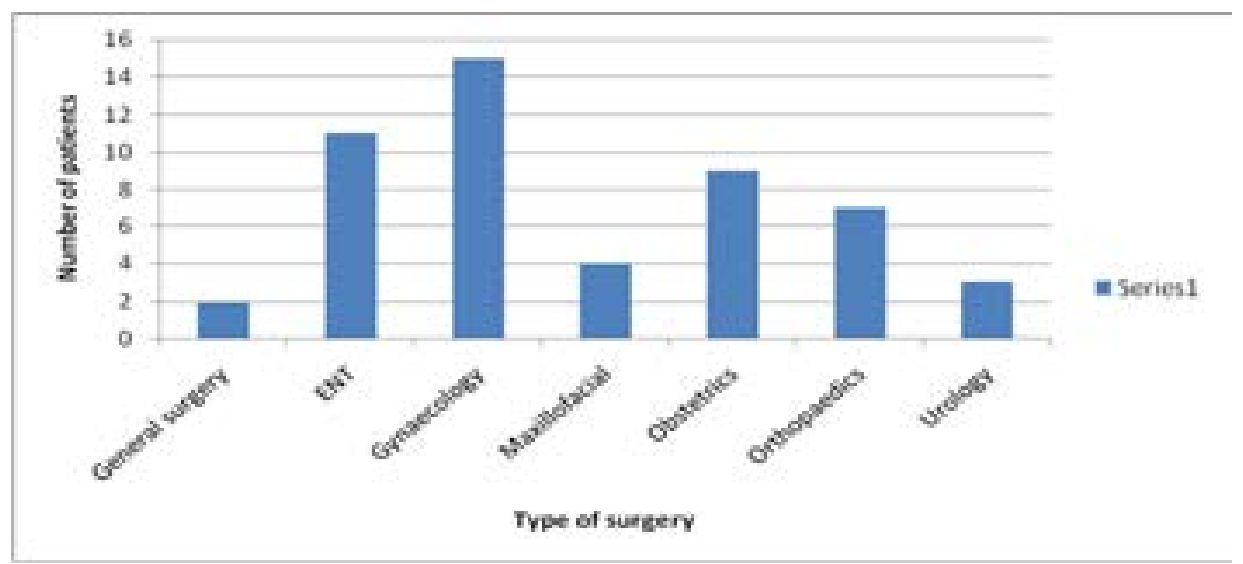

\section{Results}

A total of 51 participants were recruited into the study. Thirty eight were female $(75 \%)$. There was no significant difference in the proportions of either sex recruited in each study setting. The majority of patients, $72 \%$, were inpatients while the main indication for surgery was gynaecological. The summary of these results are presented in Table 1.

\begin{tabular}{|c|c|c|c|}
\hline & $\begin{array}{l}\text { Clinic } \\
\text { n (\%) }\end{array}$ & $\begin{array}{l}\text { Ward } \\
\text { n (\%) }\end{array}$ & Total \\
\hline \multicolumn{4}{|l|}{ Sex } \\
\hline Females & $22(58)$ & $16(42)$ & 38 \\
\hline Males & $6(46)$ & $7(54)$ & 13 \\
\hline \multicolumn{4}{|l|}{$\begin{array}{l}\text { Prior anaesthetic } \\
\text { experience }\end{array}$} \\
\hline Yes & $18(67)$ & $9(33)$ & 27 \\
\hline No & $10(42)$ & $14(58)$ & 24 \\
\hline \multicolumn{4}{|l|}{ Duration of stay } \\
\hline $\begin{array}{l}\text { Day } \\
\text { In patient }\end{array}$ & $\begin{array}{l}11(79) \\
17(46)\end{array}$ & $\begin{array}{l}3(21) \\
20(54)\end{array}$ & $\begin{array}{l}14 \\
37\end{array}$ \\
\hline Age & Clinic & Ward & Total \\
\hline $\begin{array}{l}20-24 \\
25-29 \\
30-34 \\
35-39 \\
40-44 \\
45-49 \\
50-54 \\
-59 \\
>59\end{array}$ & $\begin{array}{l}1(3.6) \\
8(28.6) \\
5(17.9) \\
4(14.3) \\
5(17.9) \\
1(3.6) \\
3(10.7) \\
1(3.6) \\
0(0)\end{array}$ & $\begin{array}{l}1(4.3) \\
3(13.0) \\
7(30.4) \\
6(26.1) \\
2(8.7) \\
1(4.3) \\
0(0) \\
2(8.7) \\
1(4.3)\end{array}$ & $\begin{array}{l}2 \\
11 \\
12 \\
10 \\
7 \\
2 \\
3 \\
3 \\
1\end{array}$ \\
\hline $\begin{array}{l}-59 \\
>59\end{array}$ & Clinic & Ward & Total \\
\hline \multicolumn{4}{|l|}{ Type of surgery } \\
\hline ENT & 2 & 0 & 2 \\
\hline General surgery & 9 & 2 & 11 \\
\hline Gynaecology & 8 & 7 & 15 \\
\hline Maxillofacial surgery & 2 & 2 & 4 \\
\hline Obstetrics & 2 & 7 & 9 \\
\hline Orthopaedics & 3 & 4 & 7 \\
\hline Urology & 2 & 1 & 3 \\
\hline
\end{tabular}


Our primary objective was to determine the difference in reduction of anxiety as measured by the change in STAI score between the clinic group and the ward group. The mean STAI score in the pre-evaluation group reviewed in the wards was 47.44 (median score 47 , range 38 to 60 ) while that of the group reviewed in the clinic was 46.86 (median score 47, range 29 to 62). The mean STAI score in the post-evaluation of the same ward group was 46.70 (median score 47 , range 37 to 62 ) while that of the clinic group was 44.71 (median score 44.5, range 29 to 58).

The mean difference in the change in score between the ward and clinic groups was 1.51 (95\% CI 1.00-2.02) with a p-value of 0.0051 indicating that there was a significant difference in the change in STAI score. Table 2 summarizes these results.

\section{Table 2: Comparison of change in anxiety score in clinic versus ward patients}

\begin{tabular}{lllll} 
Location of & $\begin{array}{l}\text { Pre- } \\
\text { anaesthesia } \\
\text { review }\end{array}$ & $\begin{array}{l}\text { Post- } \\
\text { anaesthesia } \\
\text { evaluation }\end{array}$ & $\begin{array}{l}\text { Change in } \\
\text { score }\end{array}$ & $\begin{array}{l}\text { P-value for } \\
\text { the difference } \\
\text { in change in } \\
\text { score }\end{array}$ \\
\hline Ward & 47.44 & 46.70 & 0.74 & 0.0051 \\
Clinic & 46.86 & 44.71 & 2.14 & \\
\hline
\end{tabular}

We then examined for the influences of sex, duration of hospital stay and prior anesthesia experience. Table
3 summarizes these results. There was no difference in the change in score between the groups defined by these variables.

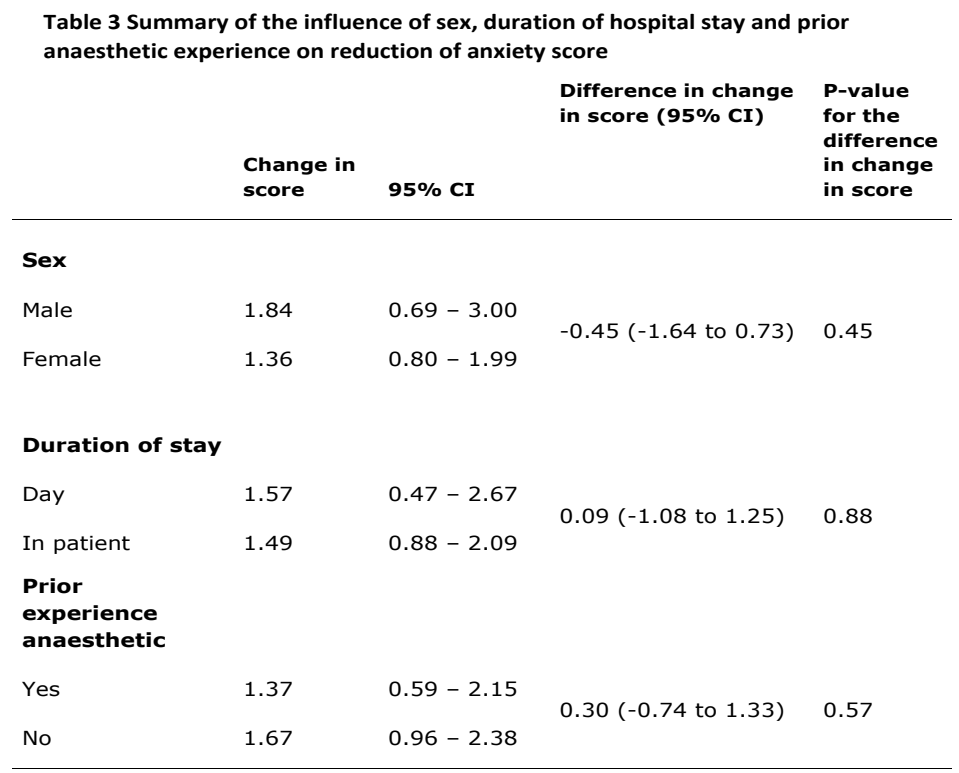

Finally, using the location of review as the explanatory variable, we determined the odds of a reduction in anxiety as measured using the STAI score as described in table 4 . The odds of a reduction in anxiety were more than 2 times higher in the group reviewed in the clinic than the group reviewed in the ward; however these odds could have been up to $30 \%$ lower or up to 7 fold higher. The study also found that there were more patients with clinically significant anxiety ${ }^{12}$ in the ward group than in the clinic group. The significance of this may be reduced due to lack of homogeneity of the population with a larger number of female patients being recruited who are known to have higher levels of anxiety. 
Table 4 Odds ratio of reduction of anxiety according to location of review

\begin{tabular}{llll}
$\begin{array}{l}\text { Location of } \\
\text { review }\end{array}$ & Odd ratio & P value & $\mathbf{9 5 \%} \mathbf{C I}$ \\
\hline Ward & 1.00 & 0.15 & $0.74-7.20$ \\
Clinic & 2.30 & & \\
\hline
\end{tabular}

\section{Discussion}

Preoperative anxiety is an important perioperative complication that the anaesthetist has to deal with. The reduction in anxiety levels following a preanaesthesia evaluation would therefore be a good measure to determine the effect of an anaesthesia evaluation depending on the timing and location of the evaluation.

In this study it was hypothesised that there would be a 10 point difference in the reduction of STAI scores pre and post anaesthesia evaluation in patients evaluated in the clinic versus those seen the ward. This 10 point difference was chosen as it represents a clinically significant reduction in the STAI scores ${ }^{12}$.

The principal finding in this study is that there is a greater reduction in the STAI scores in the patients evaluated in the clinic than those evaluated in the wards. Therefore the null hypothesis of the study was rejected. This reduction was however not as big as hypothesised. This may be due to preoperative anxiety being multi-factorial e.g. anxiety due to the surgery, anaesthesia to be administered ,uncertainty, general health, environmental factors etc ${ }^{2}$. An observational study by Jawaid et $\mathrm{al}^{15}$ found that the anxiety scores due to surgery were higher than those due to anaesthesia. The economic status of the patients may contribute largely to the overall anxiety. This is because the country has a national health provider i.e. National Hospital Insurance Fund (NHIF) which does not cover patients in private hospitals fully with most patients supplementing hospital charges with their own finances or by using private health insurance companies. The pre-anaesthesia evaluation aims to counsel the patient on anaesthetic concerns ${ }^{21}$ and due to the multifactorial nature of anxiety the effect of the preanaesthesia evaluation alone may not be adequate to cause a clinically significant reduction in anxiety scores.

There is a paucity of studies that have been done to quantify the reduction in anxiety scores after preanaesthesia evaluation in the clinic. However a study by Klopfenstein et al on 40 patients undergoing transurethral resection of the prostate(TURP) found that patients who had been evaluated in the anaesthesia clinic one to two weeks prior had less anxiety on the night of surgery. They used the MACCL score and VAS anxiety scores to measure anxiety ${ }^{19}$.

The patients were evaluated in the ward not more than 24 hours prior to surgery and were noted to have almost similar pre and post evaluation STAI scores. This finding was similar to an observational study by Lichtor et $\mathrm{al}^{22}$ on 28 patients undergoing abdominal surgery. They found that anxiety scores measured by the Profile of Mood States (POMS) were similar both in the afternoon before surgery and one hour prior to surgery. This could partly explain the reduced change in this group.

There was no significant difference in the demographic data i.e. age, sex prior anaesthetic experience between the 2 groups. There was no significant difference in the reduction of anxiety scores according to gender between the ward and clinic groups. However this study was not powered to detect a difference in the reduction of anxiety scores according to the gender of the patients.

There were 22 females and 6 males recruited in the clinic group. This could be due to the higher number of clinics associated with the female gender i.e. the gynaecology clinic and the antenatal clinic in addition to the other surgical clinics. This was also similar for patients recruited in the ward group with 16 female patients versus seven male patients being recruited. Between March 2014 and March 2015 when this study was conducted 6313 females and 3834 males underwent surgery under general anaesthesia in our operating theatres. The greater number of female patients recruited may have led to a higher anxiety score $^{3,5,15,16}$. This however did not lead to bias since it's the reduction in the anxiety scores and not the degree of anxiety that the study set out to measure.

There were more patients within the younger age groups i.e. 25-44 years as compared to above 45 years. This could have been attributed to there being more obstetrics and gynaecology patients who are commonly seen in this age bracket. The patient's ability to understand the level of the English language required to answer the questionnaire may have played a role.

African Health Sciences Vol 17 Issue 1, March, 2017 
There were no significant differences in reduction of anxiety scores between the two groups according to the duration of stay i.e. whether they were daycare patients or in-patients. However the study was not designed to detect a difference in the anxiety scores based on length of stay. There were also no significant differences in the reduction of anxiety between the two groups according to whether they had a prior anaesthetic experience or not. This study was not designed to detect a difference due to this variable.

\section{Strengths}

A reduction in the anxiety score is used as the measure of the effect of the preanaesthesia evaluation which may be more accurate than measuring the anxiety once which does not take into account baseline levels. The use of a control group to compare the effectiveness of the preanaesthesia clinic is also a strength.

\section{Limitations}

The study was not performed on a homogenous population as all patients undergoing elective non-cardiac procedures were recruited. This may result in bias since patients undergoing the different types of surgical procedures maybe associated with different levels of anxiety. The use of a measurement tool in English limited the recruited patients to those who could speak it. The other study limitation found was that the difference of a 10 point reduction chosen to represent a clinically significant reduction in anxiety, used studies based on western data and this may not be commensurate with data from the African region. AKUH is a private hospital and this would limit the generalisabilty of the findings to the rest of the Kenyan population.

\section{Conclusion}

We can conclude that the preanaesthesia clinic evaluation is more effective than preanaesthesia ward evaluation in reducing anxiety although this reduction may not be clinically significant.

\section{References}

1. Hersen M, Speilberger C, Reheiser E, Editors. Wiley. In Comprehensive Handbook of Psychological Assessment 2004: 70-74.

2. Baird WLM. Pre-operative anxiety: a study of the inci- dence and aetiology. Br J Anaesth. 1967 Jun; 39(6), 503-9 3. Kindler CH, Harms C, Amsler F, Ihde-Scholl T, Scheidegger D. The visual analog scale allows effective measurement of preoperative anxiety and detection of patients' anesthetic concerns. Anesth Analg 2000 Mar; 90(3):706-12.

4. Moerman N, Oosting H. The Amsterdam Scale (APAIS). Anaesth Analg 1996; 82: 445-51

5. Jafar MF, Khan FA. Frequency of preoperative anxiety in Pakistani surgical patients. JPMA 2009 59(6):359-63

6. Wetsch W A, Pircher I, Lederer W, Kinzl JF, Traweger C, Heinz-Erian P, et al. Preoperative stress and anxiety in day-care patients and in patients undergoing fast-track surgery. Br J Anaesth 2009 Aug; 103(2):199-205.

7. Caumo W, Schmidt a P, Schneider CN, Bergmann J, Iwamoto CW, Bandeira D, et al. Risk factors for preoperative anxiety in adults. Acta Anaesthesiol Scand 2001 Mar; 45(3):298-307.

8. Lew E, Pavlin DJ, Amundsen L. Outpatient preanaesthesia evaluation clinics. Singapore Med J 2004 Nov; 45(11):509-16.

9. Pollard JB, Olson L. Early outpatient preoperative anesthesia assessment: does it help to reduce operating room cancellations? Anesth Analg 1999 Aug; 89(2):502-5. 10. Pollard JB, Zboray AL, Mazze I. Economic benefits attributed to opening a preoperative evaluation clinic for outpatients. Anaesth Analg 1996; 83: 407-10

11. Oliver D. S Chein, The value of routine preoperative medical testing before cataract surgery $N$ Engl J of Med 2000; 342:168-75

12. Klopfenstein CE, Forster A, Gesell EV. Anesthetic assessment in an outpatient consultation clinic reduces preoperative anxiety. Can J Anaesth 2000; 47: 6:511-515

13. Evaluation P. Practice Advisory for Preanesthesia Evaluation. Anesthesiology 2002 Feb; 96(2):485-96.

14. Spielberger C. Manual for the State-Trait Anxiety Inventory. rev. ed. Consulting Psychologists Press; Palo Alto (CA): 1983

15. American Psychiatric Association Diagnostic and Statistical Manual of Mental Disorders IV, 4th Edition (2000).

16. Schneiderman N,Ironson G,Siegel S STRESS AND HEALTH: Psychological, Behavioral, and Biological Determinants. Annu Rev Clin Psychol. 2005; 1: 607-628. doi: 10.1146/annurev.clinpsy.1.102803.144141

17. Practice Advisory for Preanesthesia Evaluation. An 
Updated Report by the American Society of Anesthesiologists Task Force on Preanesthesia Evaluation. Anesthesiology 2012; 116:1-17

18. Millar K, Jelicic M, Bonke B, Asbury AJ. Assessment of preoperative anxiety: comparison of measures in patients awaiting surgery for breast cancer. BrJ Anaesth 1995 Feb; 74 (2):180-3.

19. Pritchard MJ. Identifying and assessing anxiety in pre-operative patients. Nursing standard Royal College of Nursing (Great Britain) : 2009; 23(51):35-40.

20. Jawaid M, Mushtaq A, Mukhtar S, Khan Z. Preoperative anxiety before elective surgery. JPMA 2009 59:359-63
21. Kenya demographic survey 2009 census. Population by religious affiliation. http://www.knbs.or.ke/ index.php?option $=$ com_phocadownload\&view $=$ category\&id=109:population-and-housing-census-2009\&Itemid=599

22. Lichtor JL, Johanson CE, Mhoon D,Faure EAM,Hassan SZ,Roizen MF Preoperative anxiety :does anxiety level the afternoon before surgery predict anxiety level just before surgery. Anesthesiology 1987 vol 4; 67: 594-599 23. Domar a D, Everett LL, Keller MG. Preoperative anxiety: is it a predictable entity? Anesth Analg 1989 Dec; 69(6):763-7. 